\title{
Homoclinic Solutions for a Class of Nonlinear Difference Equations
}

\author{
Ali Mai ${ }^{1,2,3}$ and Zhan Zhou ${ }^{1,2}$ \\ ${ }^{1}$ School of Mathematics and Information Science, Guangzhou University, Guangdong, Guangzhou 510006, China \\ ${ }^{2}$ Key Laboratory of Mathematics and Interdisciplinary Sciences of Guangdong Higher Education Institutes, Guangzhou University, \\ Guangdong, Guangzhou 510006, China \\ ${ }^{3}$ Department of Applied Mathematics, Yuncheng University, Shanxi, Yuncheng 044000, China
}

Correspondence should be addressed to Zhan Zhou; zzhou0321@hotmail.com

Received 11 November 2013; Accepted 6 January 2014; Published 20 February 2014

Academic Editor: Renat Zhdanov

Copyright (c) 2014 A. Mai and Z. Zhou. This is an open access article distributed under the Creative Commons Attribution License, which permits unrestricted use, distribution, and reproduction in any medium, provided the original work is properly cited.

We prove the existence of homoclinic solutions of a class of nonlinear difference equations with superlinear nonlinearity by using the generalized Nehari manifold approach. For the case where the nonlinearity is odd, we obtain infinitely many homoclinic solutions of the equations. Recent results in the literature are generalized and improved.

\section{Introduction}

In this paper, we consider the following difference equation:

$$
-\Delta u_{n}+v_{n} u_{n}-\omega u_{n}=\sigma f\left(n, u_{n}\right), \quad n \in \mathbb{Z}^{m},
$$

where $\sigma= \pm 1, \omega \in \mathbb{R}$, and

$$
\begin{aligned}
\Delta u_{n}= & u_{\left(n_{1}+1, n_{2}, \ldots, n_{m}\right)}+u_{\left(n_{1}, n_{2}+1, \ldots, n_{m}\right)}+\cdots \\
& +u_{\left(n_{1}, n_{2}, \ldots, n_{m}+1\right)}-2 m u_{\left(n_{1}, n_{2}, \ldots, n_{m}\right)} \\
& +u_{\left(n_{1}-1, n_{2}, \ldots, n_{m}\right)}+u_{\left(n_{1}, n_{2}-1, \ldots, n_{m}\right)} \\
& +\cdots+u_{\left(n_{1}, n_{2}, \ldots, n_{m}-1\right)}
\end{aligned}
$$

is the discrete Laplacian in $m$ spatial dimension.

Assume that $V=\left\{v_{n}\right\}_{n \in \mathbb{Z}^{m}}$ satisfies the following condition:

$$
\left(V_{1}\right) \quad \lim _{|n| \rightarrow \infty} v_{n}=\infty,
$$

where $|n|=\left|n_{1}\right|+\left|n_{2}\right|+\cdots+\left|n_{m}\right|$ is the length of multi-index $n$. Without loss of generality, we assume that $v_{n} \geq 1$ for all $n \in \mathbb{Z}^{m}$.
Assume further that $f(n, 0)=0$; then $u_{n} \equiv 0$ is a solution of (1), which is called the trivial solution. As usual, we call that a solution $u=\left\{u_{n}\right\}$ of (1) is homoclinic (to 0 ) if

$$
\lim _{|n| \rightarrow \infty} u_{n}=0
$$

In addition, we are interested in the existence of nontrivial homoclinic solution for (1), that is, solutions that are not equal to 0 identically. This problem appears when we look for the discrete solutions of the discrete nonlinear Schrödinger (DNLS) equation in $m$ dimensional lattices:

$$
\begin{array}{r}
i \dot{\psi}_{n}+\Delta \psi_{n}-v_{n} \psi_{n}+\sigma f\left(n, \psi_{n}\right)=0, \\
n=\left(n_{1}, n_{2}, \ldots, n_{m}\right) \in \mathbb{Z}^{m},
\end{array}
$$

where the nonlinearity $f(n, u)$ is gauge invariant; that is,

$$
f\left(n, e^{i \theta} u\right)=e^{i \theta} f(n, u), \quad \theta \in \mathbb{R} .
$$

The parameter $\sigma$ characterizes the focusing properties of the equation: if $\sigma=1$, the equation is self-focusing, while $\sigma=-1$ corresponds to the defocusing equation. 
Due to the definition of solutions, we know that $\psi_{n}$ has the form

$$
\begin{aligned}
& \psi_{n}=u_{n} e^{-i \omega t}, \\
& \lim _{|n| \rightarrow \infty} \psi_{n}=0
\end{aligned}
$$

where $\left\{u_{n}\right\}$ is a real-valued sequence and $\omega \in \mathbb{R}$ is the temporal frequency. Then (5) becomes (1) and (4) holds. Therefore, the problem on the existence of solitons of (5) has been reduced to that on the existence of solutions of the boundary value problem (1)-(4).

DNLS equation is one of the most important inherently discrete models. It plays a crucial role in the modeling of a great variety of phenomena, ranging from solid state and condensed matter physics to biology (see [1-3] and reference therein). As we know, Davydov [4] studied the discrete nonlinear Schrödinger equation in molecular biology and $\mathrm{Su}$ et al. [5] considered the equation in condensed matter physics.

The existence of discrete solutions for DNLS equations has been studied by many authors. When the potential $V=$ $\left\{v_{n}\right\}$ is unbounded, some existence results were obtained by using various methods. For example, the authors obtained the existence of discrete solutions for DNLS equations by Nehari manifold method in [6-8] and by the mountain pass theorem and fountain theorem in [9], respectively. In [10], Zhang and Pankov obtained the existence of infinitely many nontrivial solutions for DNLS equations by the linking theorem. When the potential is periodic, the existence of solutions for the periodic DNLS equations with superlinear nonlinearity [1115] and with saturable nonlinearity [16-20] has been studied, respectively.

As it is well known, the Ambrosetti-Rabinowitz condition plays a crucial role in proving the boundedness of the PalaisSmale sequence $[6,7]$. In this paper, we assume that the nonlinearity satisfies more general superlinear conditions than the classical Ambrosetti-Rabinowitz superlinearity condition $[6-8,15,21]$, and we investigate the existence and multiplicity results of homoclinic solutions for the case $\omega \in \mathbb{R}$ by the generalized Nehari manifold approach. One aim of this paper is to find ground state homoclinic solutions, that is, nontrivial homoclinic solutions corresponding to the least positive critical value of the variational functional. The other aim of this paper is to obtain sufficient conditions for the existence of infinitely many pairs of homoclinic solutions of (1).

This paper is organized as follows. The assumptions on the nonlinearity and the main results are summarized in Section 2. We mention that our results improve the corresponding results in $[6,7,9,10]$. The proofs of the main theorems are completed in Section 3.

\section{Preliminaries and Main Results}

Assume that the following conditions hold.

$$
\begin{aligned}
& \left(\mathrm{f}_{1}\right) \underset{\text { such that }}{f \in C\left(\mathbb{Z}^{m} \times \mathbb{R}, \mathbb{R}\right) \text {, and there exist } a>0, p \in(2, \infty)} \\
& \quad|f(n, u)| \leq a\left(1+|u|^{p-1}\right), \quad \forall n \in \mathbb{Z}^{m}, u \in \mathbb{R} \text {. } \\
& \left(\mathrm{f}_{2}\right) f(n,, u)=o(|u|) \text { as } u \rightarrow 0 \text { uniformly for } n \in \mathbb{Z}^{m} \text {. }
\end{aligned}
$$

$\left(\mathrm{f}_{3}\right) \lim _{|u| \rightarrow \infty} F(n, u) /|u|^{2}=+\infty$ uniformly for $n \in \mathbb{Z}^{m}$, where $F(n, u)$ is the primitive function of $f(n, u)$, that is,

$$
F(n, u)=\int_{0}^{u} f(n, s) d s
$$

$\left(\mathrm{f}_{4}\right) u \mapsto f(n, u) /|u|$ is strictly increasing on $(-\infty, 0)$ and $(0, \infty)$ for all $n \in \mathbb{Z}^{m}$.

Let

$$
\begin{aligned}
l^{p} \equiv l^{p}\left(\mathbb{Z}^{m}\right) & \\
= & \left\{u=\left\{u_{n}\right\}_{n \in \mathbb{Z}^{m}}: \forall n \in \mathbb{Z}^{m}, u_{n} \in \mathbb{R},\right. \\
& \left.\|u\|_{l^{p}}=\left(\sum_{n \in \mathbb{Z}^{m}}\left|u_{n}\right|^{p}\right)^{1 / p}<\infty\right\} .
\end{aligned}
$$

Then the following embedding between $l^{p}$ spaces holds:

$$
l^{q} \subset l^{p}, \quad\|u\|_{l^{p}} \leq\|u\|_{l^{q}}, \quad 1 \leq q \leq p \leq \infty .
$$

Let

$$
A=-\Delta+V
$$

be positive self-adjoint operator defined on $l^{2}\left(\mathbb{Z}^{m}\right)$.

Define the space

$$
E:=\left\{u \in l^{2}\left(\mathbb{Z}^{m}\right): A^{1 / 2} u \in l^{2}\left(\mathbb{Z}^{m}\right)\right\} .
$$

Then $E$ is a Hilbert space equipped with the norm

$$
\|u\|=\left\|A^{1 / 2} u\right\|_{l^{2}\left(Z^{m}\right)} .
$$

Consider the functional $J$ defined on $E$ by

$$
J(u)=\frac{1}{2}((A-\omega) u, u)-\sigma \sum_{n \in \mathbb{Z}^{m}} F\left(n, u_{n}\right) .
$$

The hypotheses on $f(n, u)$ imply that the functional $J \in$ $C^{1}(E, \mathbb{R})$. Then the derivative of $J$ has the following formula:

$$
\begin{array}{r}
\left(J^{\prime}(u), v\right)=((A-\omega) u, v)-\sigma \sum_{n \in \mathbb{Z}^{m}} f\left(n, u_{n}\right) v_{n}, \\
\forall v \in E .
\end{array}
$$

Equation (16) implies that (1) is easily recognized as the corresponding Euler-Lagrange equation for $J$. Thus, to find nontrivial solutions of (1), we need only to look for nonzero critical points of $J$.

The following lemma plays an important role in this paper; it was established in [6].

Lemma 1. If $V$ satisfies the condition $\left(V_{1}\right)$, then

(1) for any $2 \leq p \leq \infty$, the embedding map from $E$ into $l^{p}\left(\mathbb{Z}^{m}\right)$ is compact; 
(2) the spectrum $\sigma(A)$ is discrete and consists of simple eigenvalues accumulating to $+\infty$.

Let $E=E^{+} \oplus E^{0} \oplus E^{-}$, where $E^{+}, E^{0}$, and $E^{-}$correspond to the positive, zero, and negative part of the spectrum of $A-\omega$ in $E$, respectively. More precisely, by Lemma 1, we can assume that

$$
\lambda_{1} \leq \lambda_{2} \leq \cdots \leq \lambda_{k} \leq \cdots \rightarrow \infty
$$

are all eigenvalues of $A$ and a corresponding orthogonal (in $E$ ) set of eigenfunctions by $e_{1}, e_{2}, \ldots, e_{k} \ldots$. Suppose $\lambda_{k}<\omega=$ $\lambda_{k+1}=\cdots=\lambda_{m}<\lambda_{m+1}$, where $1 \leq k<m$. Then

$$
E^{-}=\operatorname{span}\left\{e_{1}, \ldots, e_{k}\right\}, \quad E^{0}=\operatorname{span}\left\{e_{k+1}, \ldots, e_{m}\right\} .
$$

We also admit the cases $k=0$ and $k=m \geq 1$ which, respectively, correspond to $E^{-}=\{0\}$ and $E^{0}=\{0\}$. For $\omega<\lambda_{1}$, we take $E^{0}=E^{-}=\{0\}$.

For any $u \in E$, letting $u=u^{+}+u^{0}+u^{-}$with $u^{ \pm} \in E^{ \pm}$, $u^{0} \in E^{0}$, we can define an equivalent norm $\|\cdot\|$ on $E^{ \pm}$by

$$
\left\|u^{ \pm}\right\|^{2}= \pm\left((A-\omega) u^{ \pm}, u^{ \pm}\right), \quad \text { for } u^{ \pm} \in E^{ \pm},
$$

respectively. So $J$ can be rewritten as

$$
J(u)=\frac{1}{2}\left\|u^{+}\right\|^{2}-\frac{1}{2}\left\|u^{-}\right\|^{2}-\sigma I(u),
$$

where $I(u)=\sum_{n \in \mathbb{Z}^{m}} F\left(n, u_{n}\right)$.

We define for $u \in E \backslash F$ the subspace

$$
E(u):=\mathbb{R} u+F=\mathbb{R} u^{+} \oplus F,
$$

and the convex subset

$$
\widehat{E}(u):=\mathbb{R}^{+} u+F=\mathbb{R}^{+} u^{+} \oplus F
$$

of $E$, where, as usual, $\mathbb{R}^{+}=[0, \infty)$ and $F=E^{0} \oplus E^{-}$. Let the generalized Nehari manifold

$$
\mathscr{M}=\left\{u \in E \backslash F: J^{\prime}(u) u=0, J^{\prime}(u) v=0 \forall v \in F\right\} .
$$

Now we are ready to state the main results.

Theorem 2. Suppose that conditions $\left(V_{1}\right),\left(f_{1}\right)-\left(f_{4}\right)$ are satisfied. Then one has the following conclusions.

(1) If $\sigma=-1, \omega \leq \lambda_{1}$, (1) has no nontrivial solution.

(2) If $\sigma=1, \omega \in \mathbb{R}$, (1) has a nontrivial ground state homoclinic solution.

Theorem 3. Suppose thatconditions $\left(V_{1}\right),\left(f_{1}\right)-\left(f_{4}\right)$ are satisfied; let $\sigma=1$ and $\omega \in \mathbb{R}$ if $f(n, u)$ is odd in $u$ for each $n \in \mathbb{Z}^{m}$. Then (1) has infinitely many pairs of homoclinic solutions $\pm u^{(k)}$ in $E$ satisfying

$$
\begin{array}{r}
\frac{1}{2}\left(A u^{(k)}, u^{(k)}\right)-\frac{1}{2} \omega\left(u^{(k)}, u^{(k)}\right)-\sum_{n \in \mathbb{Z}^{m}} F\left(n, u_{n}^{(k)}\right) \\
\longrightarrow \infty \\
\text { as } k \longrightarrow \infty .
\end{array}
$$

Remark 4. In [9], the authors considered (1); they obtained the existence of nontrivial solutions for the case $\omega<\lambda_{1}$. In our paper, we consider more general case $\omega \in \mathbb{R}$. Thus, our results extend their corresponding ones.

Remark 5. In $[6,7]$, the authors considered the following DNLS equation:

$$
-\Delta u_{n}+v_{n} u_{n}-\omega u_{n}-\sigma \gamma_{n} f\left(u_{n}\right)=0,
$$

which is a special case of (1). They obtained the existence of solutions for the case $\omega<\lambda_{1}$.

They additionally assumed that $f \in C^{1}$ and $f$ satisfies the following condition: there is a $2<q<\infty$ such that

$$
0<(q-1) f(u) u \leq f^{\prime}(u) u^{2}, \quad \forall u \neq 0,
$$

which implies

$$
0<q F(u) \leq f(u) u, \quad \forall u \neq 0 .
$$

This is the classical Ambrosetti-Rabinowitz superlinear condition. It is easy to see that (27) implies that $F(u) \geq C|u|^{q}$, for some constant $C>0$ and $|u| \geq 1$, so it is a stronger condition than $\left(f_{3}\right)$.

In [6], Zhang obtained a minimizer of the corresponding functional on the Nehari manifold $\mathcal{N}$. It is crucial to require that $f$ is of class $C^{1}$. However, in our paper, we do not assume that $f$ is of class $C^{1}$, so the generalized Nehari manifold $\mathscr{M}$ may not be a smooth manifold and it is not clear that the minimizer on $\mathscr{M}$ is a critical point of $J$. Our assumptions do not require this smoothness condition. Therefore, our results extend those of [6].

In [10], the authors considered (25) for the case $\omega \in \mathbb{R}$; they also assumed that $f \in C^{1}(\mathbb{R})$ and satisfies (27). We define $f$ by

$$
f(u)=u \ln (1+|u|)
$$

then $f$ satisfies all conditions in Theorems 2 and 3, but $f$ does not satisfy (27). Therefore, our results improve and extend their corresponding ones.

We recall some basic results from critical point theory. The following lemma plays an important role in the proof of multiplicity results. Let $S=\{w \in E:\|w\|=1\}$.

Lemma 6 (see [22]). If $E$ is a infinite-dimensional Hilbert space, $\Phi \in C^{1}(S, \mathbb{R})$ is even and bounded below and satisfies the Palais-Smale condition. Then $\Phi$ has infinitely many pairs of critical points.

\section{Proofs of Main Results}

Throughout this paper, we always assume that $\left(V_{1}\right)$ and $\left(\mathrm{f}_{1}\right)-$ $\left(\mathrm{f}_{4}\right)$ are satisfied. In this section, we consider $\sigma=1$. To continue the discussion, we need the following technical lemma. 
Lemma 7 (see [23]). Let $u, s, v \in \mathbb{R}$ be numbers with $s \geq-1$ and $w:=s u+v \neq 0$. Then

$$
\begin{aligned}
& f(n, u)\left[s\left(\frac{s}{2}+1\right) u+(1+s) v\right]+F(n, u) \\
& -F(n, u+w)<0 .
\end{aligned}
$$

Lemma 8. If $u \in \mathscr{M}$, then

$$
J(u+w)<J(u)
$$

for every $w \in U:=\{s u+v: s \geq-1, v \in F\}, w \neq 0$.

Hence $u$ is the unique global maximum of $\left.J\right|_{\widehat{E}(u)}$.

Proof. Let $B=A-\omega$; then we rewrite $J$ by

$$
J(u)=\frac{1}{2}\left(B u^{+}, u^{+}\right)+\frac{1}{2}\left(B u^{-}, u^{-}\right)-\sum_{n \in \mathbb{Z}^{m}} F\left(n, u_{n}\right) .
$$

Since $u \in \mathscr{M}$, we have

$$
\begin{aligned}
0= & \left(J^{\prime}(u), \frac{2 s+s^{2}}{2} u+(1+s) v\right) \\
= & \frac{2 s+s^{2}}{2}\left(B u^{+}, u^{+}\right)+\frac{2 s+s^{2}}{2}\left(B u^{-}, u^{-}\right) \\
& +(1+s)\left(B u^{-}, v\right) \\
& -\sum_{n \in \mathbb{Z}^{m}} f\left(n, u_{n}\right)\left(\frac{2 s+s^{2}}{2} u_{n}+(1+s) v_{n}\right) .
\end{aligned}
$$

Together with Lemma 7, we know that

$$
\begin{aligned}
& J(u+w)-J(u) \\
&= \frac{1}{2}\left\{\left(B(1+s) u^{+},(1+s) u^{+}\right)-\left(B u^{+}, u^{+}\right)\right\} \\
&+\frac{1}{2}\left\{\left(B\left((1+s) u^{-}+v\right),(1+s) u^{-}+v\right)\right. \\
&\left.\quad-\left(B u^{-}, u^{-}\right)\right\} \\
&+\sum_{n \in \mathbb{Z}^{m}} F\left(n, u_{n}\right)-\sum_{n \in \mathbb{Z}^{m}} F\left(n, u_{n}+w_{n}\right) \\
&= \frac{2 s+s^{2}}{2}\left(B u^{+}, u^{+}\right)+\frac{2 s+s^{2}}{2}\left(B u^{-}, u^{-}\right) \\
&+\frac{1}{2}(B v, v)+(1+s)\left(B u^{-}, v\right)
\end{aligned}
$$

$$
\begin{aligned}
& +\sum_{n \in \mathbb{Z}^{m}} F\left(n, u_{n}\right)-\sum_{n \in \mathbb{Z}^{m}} F\left(n, u_{n}+w_{n}\right) \\
& =\frac{1}{2}(B v, v)+\sum_{n \in \mathbb{Z}^{m}}\left\{f\left(n, u_{n}\right)\right. \\
& \times\left[s\left(\frac{s}{2}+1\right) u_{n}+(1+s) v_{n}\right] \\
& \left.+F\left(n, u_{n}\right)-F\left(n, u_{n}+w_{n}\right)\right\}<0 .
\end{aligned}
$$

The proof is complete.

Lemma 9. For each $u \in E^{+} \backslash\{0\}$, the set $\mathscr{M} \cap \widehat{E}(u)$ consists of precisely one point which is the unique global maximum of $\left.J\right|_{\widehat{E}(u)}$.

Proof. By Lemma 8 , it suffices to show that $\mathscr{M} \cap \widehat{E}(u) \neq \emptyset$. Since $\widehat{E}(u)=\widehat{E}\left(u^{+} /\left\|u^{+}\right\|\right)$, we may assume that $u \in S^{+}$. To end this proof, we should show two key conclusions.

Firstly, we claim that there exists $\alpha>0$ such that

$$
c:=\inf _{\mathscr{M}} J(u) \geq \inf _{S_{\alpha}} J(u)>0,
$$

where $S_{\alpha}:=\left\{u \in E^{+}:\|u\|=\alpha\right\}$.

In fact, by $\left(\mathrm{f}_{1}\right)$ and $\left(\mathrm{f}_{2}\right)$, it is easy to show that, for any $\varepsilon>0$, there exists $c_{\varepsilon}>0$, such that

$$
\begin{gathered}
|f(n, u)| \leq \varepsilon|u|+c_{\varepsilon}|u|^{p-1}, \\
|F(n, u)| \leq \varepsilon|u|^{2}+c_{\varepsilon}|u|^{p} .
\end{gathered}
$$

Since $\|\cdot\|$ is equivalent to the $E$ norm on $E^{+}$and $E \subset l^{q}$ for $2 \leq q \leq \infty$ with $\|u\|_{l^{q}} \leq\|u\|_{E}$, for any $\varepsilon \in(0,1 / 2)$ and $u \in E^{+}$, we have

$$
J(u) \geq \frac{1}{2}\|u\|^{2}-\varepsilon\|u\|^{2}-c_{\varepsilon}\|u\|^{p},
$$

which implies $\inf _{S_{\alpha}} J(u)>0$ for some $\alpha>0$ (small enough).

The first inequality is a consequence of Lemma 8 , since for every $u \in \mathscr{M}$ there is $s>0$ such that $s u^{+} \in \widehat{E}(u) \cap S_{\alpha}$.

Secondly, we claim that there exists $R>r>0$ such that

$$
\sup J(\partial Q) \leq 0
$$

where $Q:=\left\{u=s z_{0}+v: s \geq 0, v \in F,\|u\|<R\right\}$, for fixed $z_{0} \in E^{+}$with $\left\|z_{0}\right\|=1$.

Indeed, suppose by contradiction that there exists $u^{(k)}=$ $s^{(k)} z_{0}+u^{(k) 0}+u^{(k)-}$ such that $J\left(u^{(k)}\right)>0$ for all $k$ and $\left\|u^{(k)}\right\| \rightarrow$ $\infty$ as $k \rightarrow \infty$, where $u^{(k) 0} \in E^{0}$ and $u^{(k)-} \in E^{-}$. Set $v^{(k)}=$ $u^{(k)} /\left\|u^{(k)}\right\|=s^{(k)} z_{0}+v^{(k) 0}+v^{(k)-}$ with $v^{(k) 0} \in E^{0}$ and $v^{(k)-} \in E^{-}$; then

$$
\begin{aligned}
0<\frac{J\left(u^{(k)}\right)}{\left\|u^{(k)}\right\|^{2}}= & \frac{1}{2}\left(\left(s^{(k)}\right)^{2}-\left\|v^{(k)-}\right\|^{2}\right) \\
& -\sum_{n \in \mathbb{Z}^{m}} \frac{F\left(n, u_{n}^{(k)}\right)}{\left(u_{n}^{(k)}\right)^{2}}\left(v_{n}^{(k)}\right)^{2} .
\end{aligned}
$$


Note that, from $\left(f_{2}\right)$ and $\left(f_{4}\right)$, it is easy to get that

$$
F(n, u)>0, \quad \forall u \neq 0 .
$$

So we have

$$
\left\|v^{(k)-}\right\|^{2} \leq\left(s^{(k)}\right)^{2}=1-\left\|v^{(k) 0}\right\|^{2}-\left\|v^{(k)-}\right\|^{2} .
$$

Passing to a subsequence if necessary, we assume that $v^{(k)} \rightarrow$ $v, s^{(k)} \rightarrow s, v^{(k) 0} \rightarrow v^{0} \in E^{0}, v^{(k)-} \rightarrow v^{-} \in E^{-}$, and $v_{n}^{(k)} \rightarrow v_{n}$ for every $n$. Hence $v=s z_{0}+v^{0}+v^{-}$. We distinguish two cases to finish the proof of (37).

Case 1. If $s>0$, then there exists $n^{0} \in \mathbb{Z}^{m}$ such that $v_{n^{0}} \neq 0$, $\left|u_{n^{0}}^{(k)}\right|=\left\|u_{n^{0}}^{(k)}\right\| \cdot\left|v_{n^{0}}^{(k)}\right| \rightarrow+\infty$ as $k \rightarrow+\infty$. Then by $\left(\mathrm{f}_{3}\right)$, we have

$$
\sum_{n \in \mathbb{Z}^{m}} \frac{F\left(n, u_{n}^{(k)}\right)}{\left(u_{n}^{(k)}\right)^{2}}\left(v_{n}^{(k)}\right)^{2} \geq \frac{F\left(n, u_{n_{0}}^{(k)}\right)}{\left(u_{n_{0}}^{(k)}\right)^{2}}\left(v_{n_{0}}^{(k)}\right)^{2} \longrightarrow+\infty,
$$

which contradicts (38).

Case 2. If $s=0$, then $v^{(k)-} \rightarrow 0$ and therefore

$$
\left\|v^{(k) 0}\right\|^{2}=1-\left(s^{(k)}\right)^{2}-\left\|v^{(k)-}\right\|^{2} \longrightarrow 1 .
$$

Hence $v^{(k) 0} \rightarrow v^{0} \neq 0$ because $E^{0}$ is finite dimensional space. Consequently, $v \neq 0$ and

$$
\sum_{n \in \mathbb{Z}^{m}} \frac{F\left(n, u_{n}^{(k)}\right)}{\left(u_{n}^{(k)}\right)^{2}}\left(v_{n}^{(k)}\right)^{2} \longrightarrow+\infty,
$$

which contradicts (38). Hence, (37) holds.

By (34), we have $J(t u)>0$ for small $t>0$. Together with (37), we have

$$
0<\sup _{\widehat{E}(u)} J<\infty
$$

Finally, we show that $J$ is weakly upper semicontinuous on $\widehat{E}(u)$.

Let $v^{(k)} \rightarrow v$ in $\widehat{E}(u)$. Then $v_{n}^{(k)} \rightarrow v_{n}$ as $k \rightarrow \infty$, for all $n$ after passing to a subsequence if needed. Hence $F\left(n, v_{n}^{(k)}\right) \rightarrow$ $F\left(n, v_{n}\right)$. Then

$$
\begin{aligned}
I(v) & =\sum_{n \in \mathbb{Z}^{m}} \lim _{k \rightarrow \infty} F\left(n, v_{n}^{(k)}\right) \\
& \leq \liminf _{k \rightarrow \infty} \sum_{n \in \mathbb{Z}^{m}} F\left(n, v_{n}^{(k)}\right)=\liminf _{k \rightarrow \infty} I\left(v^{(k)}\right) ;
\end{aligned}
$$

that is, $I$ is weakly lower semicontinuous. From the weak lower semicontinuous of the norm, it is easy to see that $J$ is weakly upper semicontinuous on $\widehat{E}(u)$.

From above, we have $J\left(u_{0}\right)=\sup _{\widehat{E}(u)} J$ for some $u_{0} \in$ $\widehat{E}(u) \backslash\{0\}$. By $(37), u_{0}$ is a critical point of $\left.J\right|_{\widehat{E}(u)}$. Hence $u_{0} \in \mathscr{M}$. Consequently, $u_{0} \in \mathscr{M} \cap \widehat{E}(u)$.

This completes the proof.
Lemma 10. Suppose that conditions $\left(V_{1}\right),\left(f_{1}\right)-\left(f_{4}\right)$ are satisfied. Then $J$ satisfies the Palais-Smale condition on $\mathscr{M}$.

Proof. Suppose $\left\{u^{(k)}\right\} \subset \mathscr{M}$ is a sequence such that $J\left(u^{(k)}\right) \leq$ $d$ for some $d>0$ and $J^{\prime}\left(u^{(k)}\right) \rightarrow 0$ as $k \rightarrow \infty$.

Firstly, we prove that $\left\{u^{(k)}\right\}$ is bounded. In fact, if not, we may assume by contradiction that $\left\|u^{(k)}\right\| \rightarrow \infty$ as $k \rightarrow \infty$. Let $v^{(k)}=u^{(k)} /\left\|u^{(k)}\right\|$. Then there exists a subsequence, still denoted by the same notation, such that $v^{(k)} \rightarrow v$ in $E$ as $k \rightarrow$ $\infty$.

If $v \neq 0$, since $\left|u_{n}^{(k)}\right| \rightarrow \infty$ for some $n$, as $k \rightarrow \infty$, it follows again from $\left(\mathrm{f}_{3}\right)$ and Fatou's lemma that

$$
\begin{aligned}
0 \leq \frac{J\left(u^{(k)}\right)}{\left\|u^{(k)}\right\|^{2}}= & \frac{1}{2}\left(\left\|v^{(k)+}\right\|^{2}-\left\|v^{(k)-}\right\|^{2}\right) \\
& -\sum_{n \in \mathbb{Z}^{m}} \frac{F\left(n, u_{n}^{(k)}\right)}{\left(u_{n}^{(k)}\right)^{2}}\left(v_{n}^{(k)}\right)^{2} \longrightarrow-\infty
\end{aligned}
$$
as $k \longrightarrow \infty$

this is a contradiction. Hence $v=0$.

Note that by (39), we have

$$
\begin{aligned}
0<c \leq & \frac{1}{2}\left\|v^{(k)+}\right\|^{2}-\frac{1}{2}\left\|v^{(k)-}\right\|^{2} \\
& -\sum_{n \in \mathbb{Z}^{m}} F\left(n, v_{n}^{(k)}\right) \leq \frac{1}{2}\left(\left\|v^{(k)+}\right\|^{2}-\left\|v^{(k)-}\right\|^{2}\right) ;
\end{aligned}
$$

hence $\left\|v^{(k)+}\right\| \geq\left\|v^{(k)-}\right\|$. If $v^{(k)+} \rightarrow 0$, then $v^{(k)-} \rightarrow 0$ and

$$
\left\|v^{(k) 0}\right\|^{2}=1-\left(s^{(k)}\right)^{2}-\left\|v^{(k)-}\right\|^{2} \longrightarrow 1 \text {. }
$$

Hence $v^{(k) 0} \rightarrow v^{0} \neq 0$ because $E^{0}$ is finite dimensional space. Consequently, $v \neq 0$, a contradiction again. Therefore $v^{(k)+} \nrightarrow$ 0 ; thus there exists $\delta>0$ such that $\left\|v^{(k)+}\right\| \geq \delta$ for all $k$ after passing to a subsequence.

Since $v=0, v^{(k)+} \rightarrow 0$. Applying Lemma 1, we see that, for any $2 \leq p \leq \infty$,

$$
v^{(k)+} \longrightarrow 0 \quad \text { in } l^{p}\left(\mathbb{Z}^{m}\right) .
$$

By (35), for any $s \in \mathbb{R}$,

$$
\sum_{n \in \mathbb{Z}^{m}} F\left(n, s v_{n}^{(k)+}\right) \leq \varepsilon s^{2}\left\|v^{(k)+}\right\|_{l^{2}}^{2}+c_{\varepsilon} s^{p}\left\|v^{(k)+}\right\|_{l^{p}}^{p},
$$

which implies that $\sum_{n \in \mathbb{Z}^{m}} F\left(n, s v_{n}^{(k)+}\right) \rightarrow 0$ as $k \rightarrow \infty$.

Since $s v^{(k)+} \in \widehat{E}\left(u^{(k)}\right)$ for all $s>0$, Lemma 8 implies that

$$
\begin{aligned}
d \geq J\left(u^{(k)}\right) & \geq J\left(s v^{(k)+}\right)=\frac{s^{2}}{2}\left\|v^{(k)+}\right\|^{2}-\sum_{n \in \mathbb{Z}^{m}} F\left(n, s v_{n}^{(k)+}\right) \\
& \geq \frac{s^{2} \delta^{2}}{2}-\sum_{n \in \mathbb{Z}^{m}} F\left(n, s v_{n}^{(k)+}\right) \longrightarrow \frac{s^{2} \delta^{2}}{2}
\end{aligned}
$$


as $k \rightarrow \infty$. This is a contradiction if $s>\sqrt{2 d} / \delta$. Therefore, $\left\{u^{(k)}\right\}$ is bounded.

Finally, we show that there exists a convergent subsequence of $\left\{u^{(k)}\right\}$. Actually, there exists a subsequence, still denoted by the same notation, such that $u^{(k)}-u$. Applying Lemma 1 , we see that, for any $2 \leq q \leq \infty$,

$$
u^{(k)} \longrightarrow u \text { in } l^{q}\left(\mathbb{Z}^{m}\right) .
$$

Note that

$$
\begin{aligned}
&\left\|u^{(k)}-u\right\|^{2}-\omega\left\|u^{(k)}-u\right\|_{2}^{2} \\
&=\left(J^{\prime}\left(u^{(k)}\right)-J^{\prime}(u),\left(u^{(k)}-u\right)\right) \\
&+\sum_{n \in \mathbb{Z}^{m}}\left(f\left(n, u_{n}^{(k)}\right)-f\left(n, u_{n}\right)\right)\left(u_{n}^{(k)}-u_{n}\right) .
\end{aligned}
$$

Due to the weak convergence, it is clear that the first term $\left(J^{\prime}\left(u^{(k)}\right)-J^{\prime}(u),\left(u^{(k)}-u\right)\right) \rightarrow 0$ as $k \rightarrow \infty$. It remains to show that the second term in the right hand of equality (53) also tends to be zero as $k \rightarrow \infty$.

Indeed, according to (35) and Hölder inequality, we have

$$
\begin{gathered}
\sum_{n \in \mathbb{Z}^{m}}\left(f\left(n, u_{n}^{(k)}\right)-f\left(n, u_{n}\right)\right)\left(u_{n}^{(k)}-u_{n}\right) \\
\leq \sum_{n \in \mathbb{Z}^{m}}\left[\varepsilon\left(\left|u_{n}^{(k)}\right|+\left|u_{n}\right|\right)\right. \\
\left.+c_{\varepsilon}\left(\left|u_{n}^{(k)}\right|^{p-1}+\left|u_{n}\right|^{p-1}\right)\right]\left(u_{n}^{(k)}-u_{n}\right) \\
\leq \varepsilon\left(\left\|u^{(k)}\right\|_{l^{2}}+\|u\|_{l^{2}}\right)\left\|u^{(k)}-u\right\|_{l^{2}} \\
+c_{\varepsilon}\left(\left\|u^{(k)}\right\|_{l^{p}}^{p^{p-1}}+\|u\|_{l^{p}}^{p-1}\right)\left\|u^{(k)}-u\right\|_{l^{p}} .
\end{gathered}
$$

Therefore, combining (52) and the boundedness of $\left\{u^{(k)}\right\}$, the above inequality implies

$$
\begin{aligned}
\sum_{n \in \mathbb{Z}^{m}}\left(f\left(n, u_{n}^{(k)}\right)-f\left(n, u_{n}\right)\right)\left(u_{n}^{(k)}-u_{n}\right) & \longrightarrow 0 \\
\text { as } k & \longrightarrow \infty .
\end{aligned}
$$

It follows from (53) that $u^{(k)} \rightarrow u$ inE, and this means that $J$ satisfies Palais-Smale condition. The proof is complete.

Proof of Theorem 2. (1) If $\sigma=-1$ and $\omega \leq \lambda_{1}$, we suppose that (1) has a nontrivial solution $u \in E$. Then $u$ is a nonzero critical point of $J$ in $E$ and $J^{\prime}(u)=0$. But

$$
\begin{aligned}
\left(J^{\prime}(u), u\right) & =((L-\omega) u, u)+\sum_{n \in \mathbb{Z}^{m}} f\left(n, u_{n}\right) u_{n} \\
& \geq \sum_{n \in \mathbb{Z}^{m}} f\left(n, u_{n}\right) u_{n}>0 .
\end{aligned}
$$

This is a contradiction.

(2) Let $\sigma=1$ and $\omega \in \mathbb{R}$. Suppose that conditions $\left(V_{1}\right),\left(f_{1}\right)-\left(f_{4}\right)$ are satisfied; then $\mathscr{M}$ may not be of class of
$C^{1}$; nevertheless, $\mathscr{M}$ is still a topological manifold, naturally homeomorphic to the unit sphere in $E^{+}$. So, we may define a homeomorphism between $S^{+}$and $\mathscr{M}$, where $S^{+}:=\{u \in$ $\left.E^{+}:\|u\|=1\right\}$. We distinguish five steps to end this proof of Theorem 2 .

Step 1. We define a homeomorphism between $S^{+}$and $\mathscr{M}$.

According to Lemma 9, for each $u \in E^{+} \backslash\{0\}$, we may define mapping

$$
\begin{gathered}
\widehat{m}: E^{+} \backslash\{0\} \longrightarrow \mathscr{M}, \\
u \longmapsto \widehat{m}(u),
\end{gathered}
$$

where $\widehat{m}(u)$ is the unique point of $\mathscr{M} \cap \widehat{E}(u)$. Then mapping $\widehat{m}: E^{+} \backslash\{0\} \rightarrow \mathscr{M}$ is continuous.

In fact, let $\left(u^{(k)}\right) \subset E^{+} \backslash\{0\}$ be a sequence with $u^{(k)} \rightarrow$ $u$. Since $\widehat{m}(w)=\widehat{m}\left(w^{+} /\left\|w^{+}\right\|\right)$, without loss of generality, we may assume that $\left\|u^{(k)}\right\|=1$ for all $k$. Then $\widehat{m}\left(u^{(k)}\right)=$ $\left\|\widehat{m}\left(u^{(k)}\right)^{+}\right\| u^{(k)}+v^{(k)}$, where $v^{(k)}=\widehat{m}\left(u^{(k)}\right)^{0}+\widehat{m}\left(u^{(k)}\right)^{-} \in F$. By (37) there exists $R>0$ such that

$$
\begin{aligned}
J\left(\widehat{m}\left(u^{(k)}\right)\right) & =\sup _{E\left(u^{(k)}\right)} J \leq \sup _{B_{R}(0)} J \\
& \leq \sup _{u \in B_{R}(0)}\left\|u^{+}\right\|^{2}=R^{2} \quad \text { for every } k .
\end{aligned}
$$

It follows from Lemma 10 that $\widehat{m}\left(u^{(k)}\right)$ is bounded. Passing to a subsequence if needed, we may assume that

$$
\begin{gathered}
t^{(k)}:=\left\|\widehat{m}\left(u^{(k)}\right)^{+}\right\| \longrightarrow t, \\
v^{(k)} \rightarrow v_{*}=v_{*}^{0}+v_{*}^{-} \quad \text { in } \quad E \text { as } k \longrightarrow \infty,
\end{gathered}
$$

where $t \geq \sqrt{2 c}>0$ by (47). Let $\widehat{m}(u)=t u+v$. Moreover, by Lemma 9,

$$
\begin{aligned}
J\left(\widehat{m}\left(u^{(k)}\right)\right) \geq & J\left(t^{(k)} u^{(k)}+v^{(k)}\right) \\
& \longrightarrow J\left(t u+\widehat{m}(u)^{-}\right)=J(\widehat{m}(u)) .
\end{aligned}
$$

Therefore, using the weak lower semicontinuity of the norm and $I$, we get

$$
\begin{aligned}
J(\widehat{m}(u)) & \leq \lim _{k \rightarrow \infty} J\left(\widehat{m}\left(u^{(k)}\right)\right) \\
& =\lim _{k \rightarrow \infty}\left(\frac{1}{2}\left(t^{(k)}\right)^{2}-\frac{1}{2}\left\|\widehat{m}\left(u^{(k)}\right)^{-}\right\|^{2}-I\left(\widehat{m}\left(u_{n}^{(k)}\right)\right)\right) \\
& \leq \frac{1}{2} t^{2}-\frac{1}{2}\left\|v_{*}^{-}\right\|^{2}-I\left(t u_{n}+v_{*}\right) \\
& =J\left(t u+v_{*}\right) \leq J(\widehat{m}(u)) .
\end{aligned}
$$

Hence all inequalities above must be equalities and it follows that $\widehat{m}\left(u^{(k)}\right)^{-} \rightarrow v_{*}^{-}$and $\widehat{m}\left(u^{(k)}\right)^{0} \rightarrow v_{*}^{0}$. By Lemma $9, v_{*}=v$ and hence $\widehat{m}\left(u^{(k)}\right) \rightarrow \widehat{m}(u)$. 
Next, we define mapping

$$
m=\left.\widehat{m}\right|_{S^{+}}: S^{+} \longrightarrow \mathscr{M}
$$

and the inverse of $m$ is given by $m^{-1}(u)=u^{+} /\left\|u^{+}\right\|$. It is easy to show that $m$ is a homeomorphism between $S^{+}$and $\mathscr{M}$ from above.

Step 2. We consider the functional $\widehat{\Psi}: E^{+} \backslash\{0\} \rightarrow \mathbb{R}$ and $\Psi: S^{+} \rightarrow \mathbb{R}$ defined by

$$
\widehat{\Psi}:=J(\widehat{m}(w)), \quad \Psi:=\left.\widehat{\Psi}\right|_{S^{+}} .
$$

Then $\widehat{\Psi} \in C^{1}\left(E^{+} \backslash\{0\}, \mathbb{R}\right)$ and

$$
\widehat{\Psi}^{\prime}(w) z=\frac{\left\|\widehat{m}(w)^{+}\right\|}{\|w\|} J^{\prime}(\widehat{m}(w)) z \quad \forall w, z \in E^{+}, w \neq 0 .
$$

Moreover, $\Psi \in C^{1}\left(S^{+}, \mathbb{R}\right)$ and

$$
\begin{aligned}
\Psi^{\prime}(w) z & =\left\|m(w)^{+}\right\| J^{\prime}(m(w)) z \\
\forall z \in T_{w}\left(S^{+}\right) & =\left\{v \in E^{+}:(w, v)=0\right\} .
\end{aligned}
$$

In fact, we put $u=\widehat{m}(w) \in \mathscr{M}$, so we have $u=\left(\left\|u^{+}\right\| /\right.$ $\|w\|) w+u^{-}$. Let $z \in E^{+}$. Choose $\delta>0$ such that $w_{t}:=w+$ $t z \in E^{+} \backslash\{0\}$ for $|t|<\delta$ and put $u_{t}=\widehat{m}\left(w_{t}\right) \in \mathscr{M}$. We may write $u_{t}=s_{t} w_{t}+u_{t}^{-}$with $s_{t}>0$. From above, we know that the function $t \mapsto s_{t}$ is continuous. Then $s_{0}=\left\|u^{+}\right\| /\|w\|$. By Lemma 9 and the mean value theorem, we have

$$
\begin{aligned}
\widehat{\Psi}\left(w_{t}\right)-\widehat{\Psi}(w) & =J\left(u_{t}\right)-J(u) \\
& =J\left(s_{t} w_{t}+u_{t}^{-}\right)-J\left(s_{0} w+u^{-}\right) \\
& \leq J\left(s_{t} w_{t}+u_{t}^{-}\right)-J\left(s_{t} w+u_{t}^{-}\right) \\
& =J^{\prime}\left(s_{t}\left[w+\eta_{t}\left(w_{t}-w\right)\right]+u_{t}^{-}\right) s_{t} t z,
\end{aligned}
$$

with some $\eta_{t} \in(0,1)$. Similarly,

$$
\begin{aligned}
\widehat{\Psi}\left(w_{t}\right)-\widehat{\Psi}(w) & =J\left(s_{t} w_{t}+u_{t}^{-}\right)-J\left(s_{0} w+u^{-}\right) \\
& \geq J\left(s_{0} w_{t}+u^{-}\right)-J\left(s_{0} w+u^{-}\right) \\
& =J^{\prime}\left(s_{0}\left[w+\tau_{t}\left(w_{t}-w\right)\right]+u^{-}\right) s_{0} t z
\end{aligned}
$$

with some $\tau_{t} \in(0,1)$. Combining these inequalities and the continuity of function $t \mapsto s_{t}$, we have

$$
\lim _{t \rightarrow 0} \frac{\widehat{\Psi}\left(w_{t}\right)-\widehat{\Psi}(w)}{t}=s_{0} J^{\prime}(u) z=\frac{\left\|\widehat{m}(w)^{+}\right\|}{\|w\|} J^{\prime}(\widehat{m}(w)) z .
$$

Hence the Gâteaux derivative of $\widehat{\Psi}$ is bounded linear in $z$ and continuous in $w$. It follows that $\widehat{\Psi}$ is of class $C^{1}$ (see [21]).

Note only that since $w \in S^{+}$, we have $m(w)=\widehat{m}(w)$, so (65) holds.

Step 3. We will show that $\left\{w_{n}\right\}$ is a Palais-Smale sequence for $\Psi$ if and only if $\left\{m\left(w_{n}\right)\right\}$ is a Palais-Smale sequence for $J$.
Indeed, let $\left\{w_{n}\right\}$ be a Palais-Smale sequence for $\Psi$, and let $u_{n}=m\left(w_{n}\right) \in \mathscr{M}$. Since for every $n \in \mathbb{Z}$ we have an orthogonal splitting $E=T_{w_{n}} S^{+} \oplus E\left(w_{n}\right)$; by Step 2, we have

$$
\begin{aligned}
\left\|\Psi^{\prime}\left(w_{n}\right)\right\| & =\sup _{\substack{z \in T_{w_{n}} S^{+} \\
\|z\|=1}} \Psi^{\prime}\left(w_{n}\right) z \\
& =\left\|m\left(w_{n}\right)^{+}\right\| \sup _{\substack{z \in T_{w_{n}} s^{+} \\
\|z\|=1}} J^{\prime}\left(m\left(w_{n}\right)\right) z \\
& =\left\|u_{n}^{+}\right\| \sup _{\substack{z \in T_{w_{n}} \\
\|z\|=1}} J^{\prime}\left(u_{n}\right) z,
\end{aligned}
$$

because $J^{\prime}\left(u_{n}\right) v=0$ for all $v \in E\left(w_{n}\right)$ and $E\left(w_{n}\right)$ is orthogonal to $T_{w_{n}} S^{+}$. Then

$$
\begin{aligned}
\left\|\Psi^{\prime}\left(w_{n}\right)\right\| & \leq\left\|u_{n}^{+}\right\|\left\|J^{\prime}\left(u_{n}\right)\right\| \\
& =\left\|u_{n}^{+}\right\| \sup _{\substack{z \in T_{w_{n}} S^{+}, v \in E\left(w_{n}\right) \\
z+v \neq 0}} \frac{J^{\prime}\left(u_{n}\right)(z+v)}{\|z+v\|} \\
& \leq\left\|u_{n}^{+}\right\| \sup _{z \in T_{w_{n}} S^{+} \backslash\{0\}} \frac{J^{\prime}\left(u_{n}\right)(z)}{\|z\|}=\left\|\Psi^{\prime}\left(w_{n}\right)\right\| .
\end{aligned}
$$

Therefore

$$
\left\|\Psi^{\prime}\left(w_{n}\right)\right\|=\left\|u_{n}^{+}\right\|\left\|J^{\prime}\left(u_{n}\right)\right\| .
$$

According to (47) and Lemma 10, $\sqrt{2 c} \leq\left\|u_{n}^{+}\right\| \leq \sup _{n}\left\|u_{n}^{+}\right\|<$ $\infty$. Hence $\left\{w_{n}\right\}$ is a Palais-Smale sequence for $\Psi$ if and only if $\left\{u_{n}\right\}$ is a Palais-Smale sequence for $J$.

Step 4. By (71), $\Psi^{\prime}(w)=0$ if and only if $J^{\prime}(m(w))=0$. Obviously, we have $w \in S^{+}$is a critical point of $\Psi$ if and only if $m(w) \in \mathscr{M}$ is a nontrivial critical point of $J$. Moreover, the corresponding values of $\Psi$ and $J$ coincide and $\inf _{S^{+}} \Psi=$ $\inf _{\mathscr{M}} J=c$.

Step 5. We claim that $\Psi$ satisfies the Palais-Smale condition.

Let $\left\{w^{(k)}\right\}$ be a Palais-Smale sequence for $\Psi$; it follows from Step 3 that $\left\{u^{(k)}\right\}$ is a Palais-Smale sequence for $J$, where $u^{(k)}:=m\left(w^{(k)}\right) \in \mathscr{M}$. By Lemma 10, we have $u^{(k)} \rightarrow u$ after passing to a subsequence and $w^{(k)} \rightarrow m^{-1}(u)$; this implies that $\left\{w^{(k)}\right\}$ has a convergent subsequence. Therefore, $\Psi$ satisfies the Palais-Smale condition.

Finally, let $\left\{w^{(k)}\right\} \subset S^{+}$be a minimizing sequence for $\Psi$. By Ekeland's variational principle we may assume $\Psi^{\prime}\left(w^{(k)}\right) \rightarrow 0$ as $k \rightarrow \infty$; then $\left\{w^{(k)}\right\}$ is a Palais-Smale sequence for $\Psi$. By Palais-Smale condition, $\left\{w^{(k)}\right\}$ has a convergent subsequence, still denoted by $w^{(k)}$ such that $w^{(k)} \rightarrow w$. Hence $w$ is a minimizer for $\Psi$ and therefore a critical point of $\Psi$; then $u=m(w)$ is a critical point of $J$ and also is a minimizer for $J$. That is, $u$ is a ground state homoclinic solution of (1).

This completes Theorem 2. 
Proof of Theorem 3. If $f$ is odd in $u$ for each $n \in \mathbb{Z}^{m}$, then $J$ is even, so is $\Psi$. Since $\inf _{S^{+}} \Psi=\inf _{\mathscr{M}} J>0$ by (34), $\Psi$ is bounded from below. Note that $\Psi$ satisfies the Palais-Smale condition in proof of Theorem 2. This combining with Lemma 6, $\Psi$ has infinitely many pairs of critical points. Consequently, (1) has infinitely many pairs of solutions $\pm u^{(k)}$ in $E$.

Let

$$
\begin{gathered}
\Gamma_{k}:=\left\{A \subset S^{+}: A=-A, A \text { is compact and } \gamma(A) \geq k\right\}, \\
\mathcal{c}_{k}=\inf _{A \in \Gamma_{k w \in A}} \sup \Psi(w), \quad k=1,2, \ldots,
\end{gathered}
$$

where $\gamma$ denotes the usual Kranoselskii genus ([22]). Since $\inf _{S^{+}} \Psi>0,0<c_{1} \leq c_{2} \leq \ldots$ for all $k$. Now standard arguments using the deformation lemma imply that all $c_{k}$ are critical values of $\Psi$ and $c_{k} \rightarrow \infty$; that is,

$$
\begin{aligned}
c_{k}=\Psi\left(w^{(k)}\right)= & J\left(u^{(k)}\right) \\
= & \frac{1}{2}\left(A u^{(k)}, u^{(k)}\right)-\frac{1}{2} \omega\left(u^{(k)}, u^{(k)}\right) \\
& -\sum_{n \in \mathbb{Z}^{m}} F\left(n, u_{n}^{(k)}\right) \longrightarrow \infty,
\end{aligned}
$$

as $k \rightarrow \infty$.

This completes Theorem 3.

\section{Conflict of Interests}

The authors declare that there is no conflict of interests regarding the publication of this paper.

\section{Acknowledgments}

The authors would like to thank the anonymous referee for his/her valuable suggestions. This work is supported by Program for Changjiang Scholars and Innovative Research Team in University (no. IRT1226), the National Natural Science Foundation of China (no. 11171078), the Specialized Fund for the Doctoral Program of Higher Education of China (no. 20114410110002), and the Project for High Level Talents of Guangdong Higher Education Institutes.

\section{References}

[1] S. Flach and A. Gorbach, "Discrete breakers-advances in theory and applications," Physics Reports, vol. 467, pp. 1-116, 2008.

[2] S. Flach and C. R. Willis, "Discrete breathers," Physics Reports, vol. 295, no. 5, pp. 181-264, 1998.

[3] D. Hennig and G. P. Tsironis, "Wave transmission in nonlinear lattices," Physics Reports, vol. 307, no. 5-6, pp. 333-432, 1999.

[4] A. Davydov, "The theory of contraction of proteins under their excitation," Journal of Theoretical Biology, vol. 38, pp. 559-569, 1973.

[5] W. Su, J. Schieffer, and A. Heeger, "Solions in polyacetylene," Physical Review Letters, vol. 42, pp. 1698-1701, 1979.

[6] G. Zhang, "Breather solutions of the discrete nonlinear Schrödinger equations with unbounded potentials," Journal of Mathematical Physics, vol. 50, no. 1, Article ID 013505, 2009.
[7] G. Zhang and F. Liu, "Existence of breather solutions of the DNLS equations with unbounded potentials," Nonlinear Analysis. Theory, Methods \& Applications A, vol. 71, no. 12, pp. e786-e792, 2009.

[8] G. Zhang and A. Pankov, "Standing waves of the discrete nonlinear Schrödinger equations with growing potentials," Communications in Mathematical Analysis, vol. 5, no. 2, pp. 38-49, 2008.

[9] D. Ma and Z. Zhou, "Existence and multiplicity results of homoclinic solutions for the DNLS equations with unbounded potentials," Abstract and Applied Analysis, vol. 2012, Article ID 703596, 15 pages, 2012.

[10] G. Zhang and A. Pankov, "Standing wave solutions of the discrete non-linear Schrödinger equations with unbounded potentials. II," Applicable Analysis, vol. 89, no. 9, pp. 1541-1557, 2010.

[11] G. Chen and S. Ma, "Discrete nonlinear Schrödinger equations with superlinear nonlinearities," Applied Mathematics and Computation, vol. 218, no. 9, pp. 5496-5507, 2012.

[12] A. Mai and Z. Zhou, "Ground state solutions for the periodic discrete nonlinear Schrödinger equations with superlinear nonlinearities," Abstract and Applied Analysis, vol. 2013, Article ID 317139, 11 pages, 2013.

[13] A. Mai and Z. Zhou, "Discrete solitons for periodic discrete nonlinear Schrödinger equations," Applied Mathematics and Computation, vol. 222, pp. 34-41, 2013.

[14] A. Pankov, "Gap solitons in periodic discrete nonlinear Schrödinger equations," Nonlinearity, vol. 19, no. 1, pp. 27-40, 2006.

[15] A. Pankov, "Gap solitons in periodic discrete nonlinear Schrödinger equations. II. A generalized Nehari manifold approach," Discrete and Continuous Dynamical Systems A, vol. 19, no. 2, pp. 419-430, 2007.

[16] H. Shi, "Gap solitons in periodic discrete Schrödinger equations with nonlinearity," Acta Applicandae Mathematicae, vol. 109, no. 3, pp. 1065-1075, 2010.

[17] H. Shi and H. Zhang, "Existence of gap solitons in periodic discrete nonlinear Schrödinger equations," Journal of Mathematical Analysis and Applications, vol. 361, no. 2, pp. 411-419, 2010.

[18] Z. Zhou and J. Yu, "On the existence of homoclinic solutions of a class of discrete nonlinear periodic systems," Journal of Differential Equations, vol. 249, no. 5, pp. 1199-1212, 2010.

[19] Z. Zhou, J. Yu, and Y. Chen, "On the existence of gap solitons in a periodic discrete nonlinear Schrödinger equation with saturable nonlinearity," Nonlinearity, vol. 23, no. 7, pp. 17271740, 2010.

[20] Z. Zhou, J. Yu, and Y. Chen, "Homoclinic solutions in periodic difference equations with saturable nonlinearity," Science China, vol. 54, no. 1, pp. 83-93, 2011.

[21] M. Willem, Minimax Theorems, Birkhäuser, Boston, Mass, USA, 1996.

[22] P. H. Rabinowitz, Minimax Methods in Critical Point Theory with Applications to Differential Equations, vol. 65 of CBMS Regional Conference Series in Mathematics, American Mathematical Society, Providence, RI, USA, 1986.

[23] A. Szulkin and T. Weth, "The method of Nehari manifold," in Handbook of Nonconvex Analysis and Applications, D. Y. Gao and D. Motreanu, Eds., pp. 597-632, International Press, Boston, Mass, USA, 2010. 


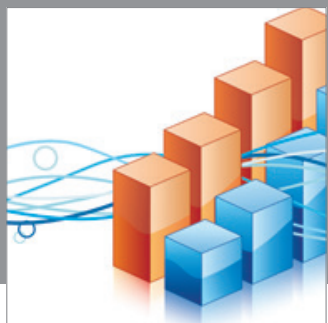

Advances in

Operations Research

mansans

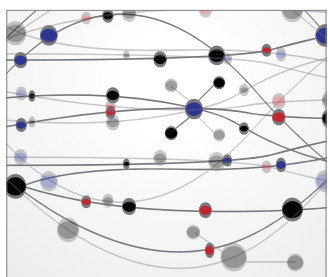

The Scientific World Journal
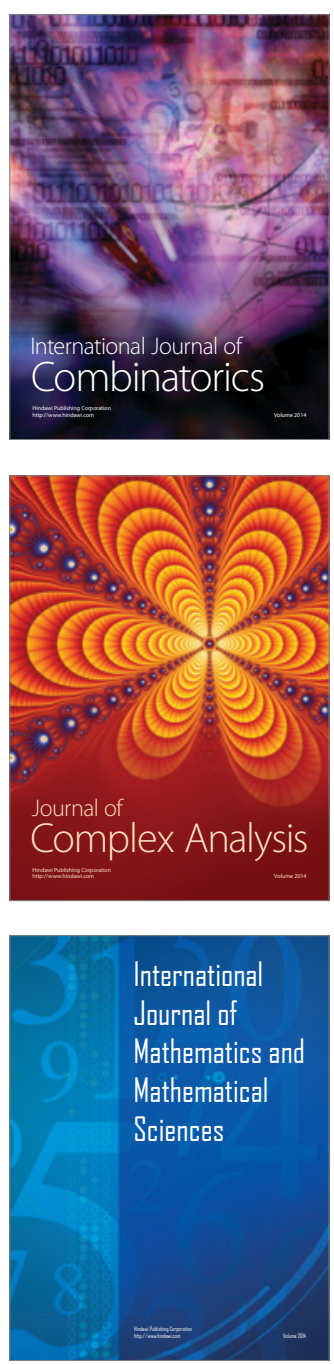
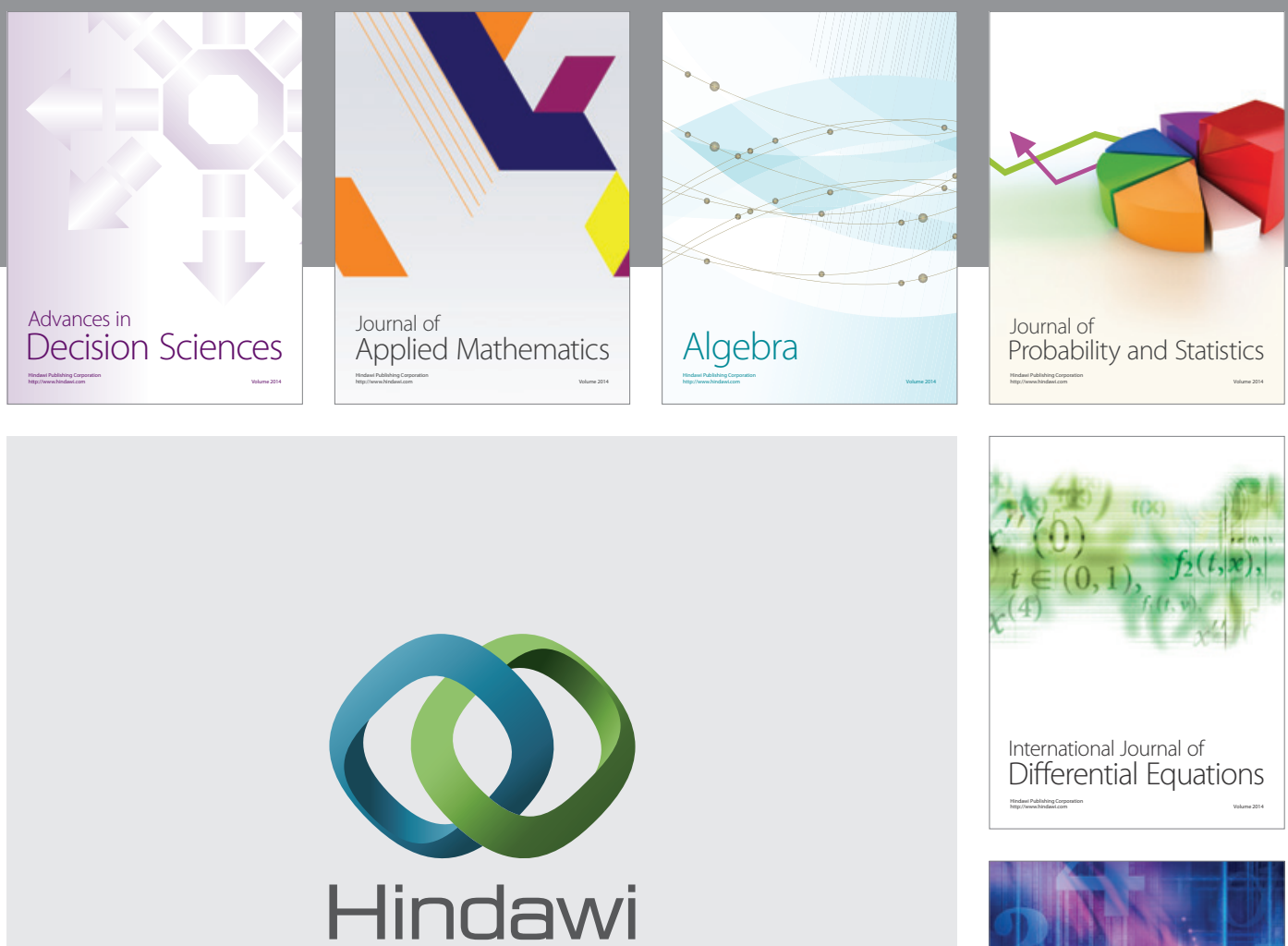

Submit your manuscripts at http://www.hindawi.com
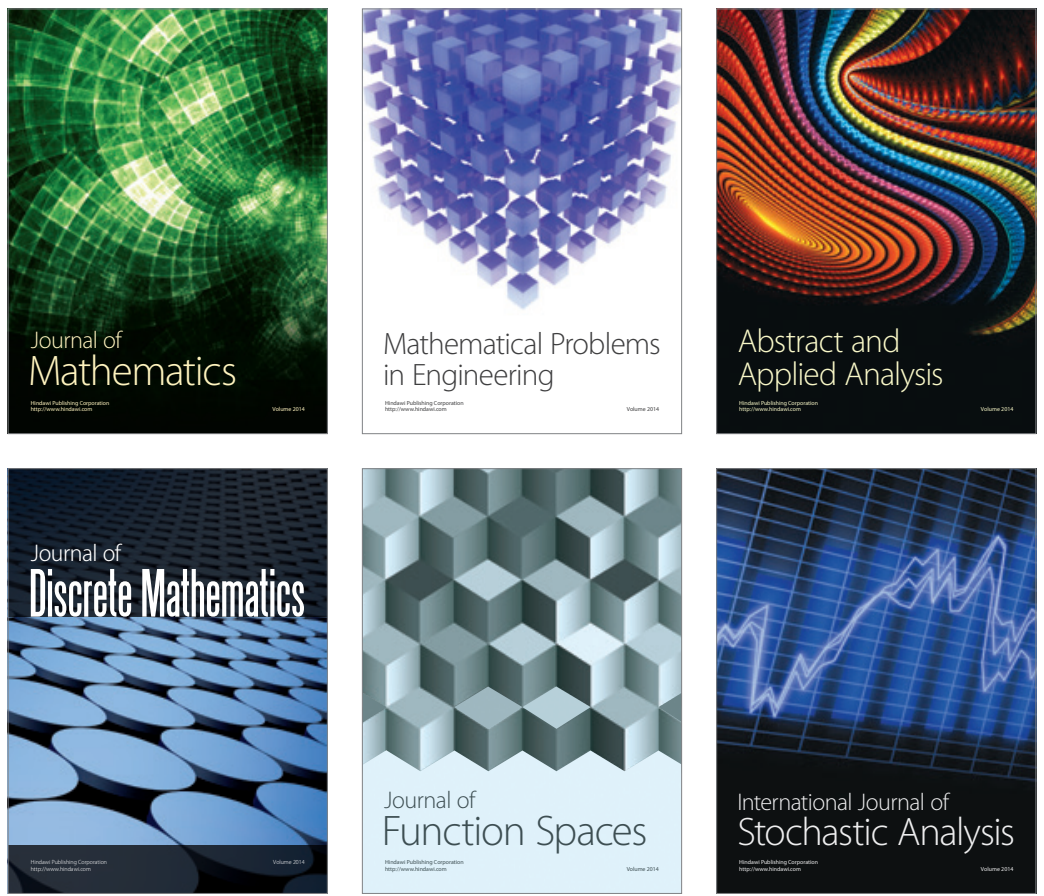

Journal of

Function Spaces

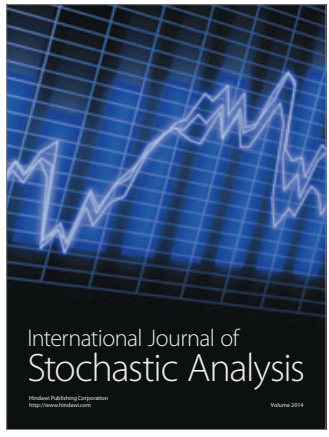

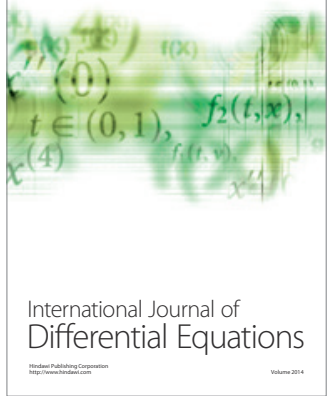
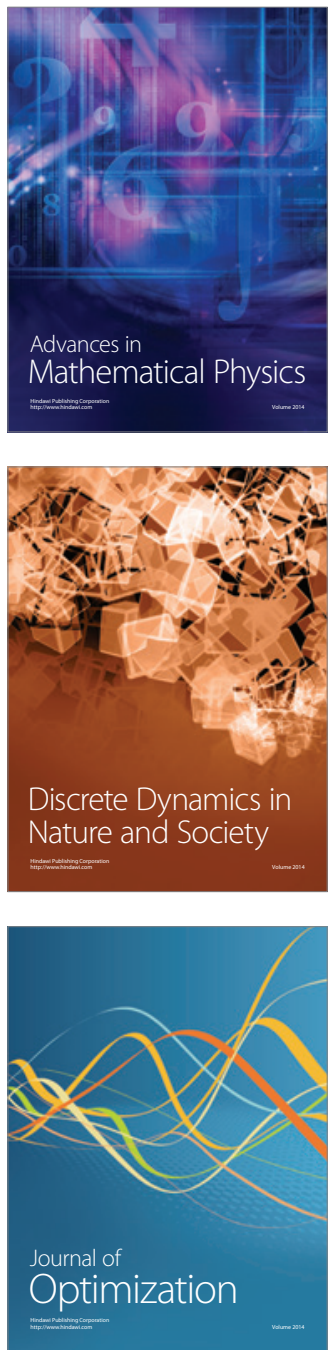